\title{
Occupation numbers in strongly polarized Fermi gases and the Luttinger theorem
}

\author{
Michael Urban ${ }^{1}$ and Peter Schuck ${ }^{1,2}$ \\ ${ }^{1}$ Institut de Physique Nucléaire, CNRS-IN2P3 and Université Paris-Sud, 91406 Orsay Cedex, France \\ ${ }^{2}$ Laboratoire de Physique et Modélisation des Milieux Condensés, \\ CNRS and Université Joseph Fourier, Maison des Magistères, BP 166, 38042 Grenoble Cedex, France
}

\begin{abstract}
We study a two-component Fermi gas that is so strongly polarized that it remains normal fluid at zero temperature. We calculate the occupation numbers within the particle-particle random-phase approximation, which is similar to the Nozières-Schmitt-Rink approach. We show that the Luttinger theorem is fulfilled in this approach. We also study the change of the chemical potentials which allows us to extract, in the limit of extreme polarization, the polaron energy.
\end{abstract}

PACS numbers: 03.75.Ss,67.85.Lm

\section{NOZIÈRES-SCHMITT-RINK APPROACH AND THE PARTICLE-PARTICLE RANDOM-PHASE APPROXIMATION}

Already many years ago, the possibility of a crossover from Cooper pairs in the Bardeen-Cooper-Schrieffer (BCS) state to a Bose-Einstein condensate (BEC) of molecules was discussed theoretically [1, 2]. Later, Nozières and Schmitt-Rink (NSR) developed a theory that correctly interpolates between the critical temperatures in the two limits [3]. In this theory, only equal densities of the two species forming the pairs (which we will denote by spin indices $\sigma=\uparrow, \downarrow$ ) were considered. Nowadays, the crossover can be realized in experiments with ultracold trapped atoms whose scattering length $a$ can be tuned with the help of a Feshbach resonance [4]. In these experiments, it is also possible to study systems with different densities of the two species, $n_{\uparrow} \neq n_{\downarrow}[5]$. In this way, one tries to discover, e.g., phases with exotic pairing, like the Fulde-Ferrel-Larkin-Ovchinnikov (FFLO) phase [6, 7]. Also the extremely polarized case, where only a single particle of spin $\downarrow$ is put into a Fermi sea of spin $\uparrow$, bears interesting physics: when passing though the Feshbach resonance from the attractive $(a<0)$ to the repulsive $(a>0)$ side, one expects that the ground state transforms from a Fermi sea plus a fermionic quasiparticle, the so-called polaron, into a Fermi sea plus a bosonic molecule [8]. To our knowledge, there is so far no unique many-body theory that can describe the imbalanced Fermi gas in the cross-over and that reproduces in the limit of extreme polarization the polaronic and the molecular ground state, depending on the value of the scattering length $a$.

As mentioned before, the original NSR theory was formulated in order to describe the BEC-BCS crossover in a two-component $(\sigma=\uparrow, \downarrow)$ Fermi gas with equal populations. Within this approach, the critical temperature $T_{c}$ as a function of the chemical potential $\mu$ is obtained from the Thouless criterion, i.e., it is the temperature where the in-medium $\mathrm{T}$ matrix develops a pole,

$$
\Gamma^{-1}(\omega=0, \mathbf{k}=0)=0
$$

where $\omega$ and $\mathbf{k}$ are, respectively, the total energy (measured from $2 \mu$ ) and momentum of the pair, and $\Gamma$ is ob-

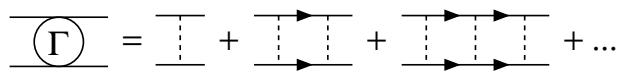

FIG. 1: Feynman diagrams for the $\mathrm{T}$ matrix in ladder approximation.

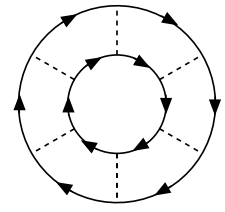

(a)

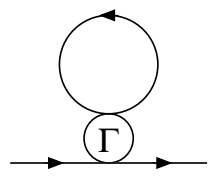

(b)
FIG. 2: (a) Typical diagram for the thermodynamic potential in the NSR approach. (b) Self-energy included to first order in the calculation of the correlated density.

tained by summing ladder diagrams, see Fig. 1. As a function of $\mu$, the critical temperature obtained in this way is exactly the same as within BCS theory. The difference between BCS and NSR comes from the inclusion of pair correlations into the relationship $n(\mu)$ between the number density and the chemical potential. This is done by including diagrams of the type shown in Fig. 2(a) into the thermodynamic potential $\Omega(\mu, T)$ and then computing the density from $n=-\partial \Omega / \partial \mu$. This is equivalent to calculating the density from [9]

$$
n=2 T \int \frac{d^{3} p}{(2 \pi)^{3}} \sum_{n \text { odd }} e^{i \omega_{n} \eta} G\left(i \omega_{n}, \mathbf{p}\right)
$$

where $\omega_{n}=n \pi T$ is a fermionic ( $n$ odd) Matsubara frequency and $G$ is the single-particle (s.p.) Green's function with at most one self-energy insertion,

$$
G=G_{0}+G_{0}^{2} \Sigma,
$$

as displayed in Fig. 2(b). Notice that in the literature one can also find variants of the NSR scheme where the Dyson series for $G$ is summed to all orders, e.g., Ref. [10].

Before turning to the imbalanced case, we want to discuss in some detail the relation between the NSR scheme 
and the random-phase approximation (RPA), here in the so-called particle-particle (pp) channel (pp-RPA) 11] in contrast to the more familiar particle-hole (ph) channel (ph-RPA). While the latter consists in the resummation of "bubble diagrams", the pp-RPA consists in a resummation of ladder diagrams as shown in Fig. 1. In both ph- and pp-RPA, the lines correspond to the propagators obtained at the Hartree-Fock (HF) level. At $T=0$, which is the case we consider in the present work, the propagators are chronological ones and belong to a fixed Fermi momentum $k_{F}$ and not to a fixed chemical potential $\mu$. The RPA correlation energy (i.e., correction to the HF energy) can be obtained from the usual couplingconstant integration [9], where one integrates, however, only over the coupling constant appearing explicitly in the bubble or ladder diagrams, respectively, keeping the HF field fixed. The formula (3) for the Green's function to be used, e.g., in the calculation of the correlation energy has to be slightly modified: now $G_{0}$ denotes the HF Green's function, and $\Sigma$ is the self-energy without the HF field.

In practice, the subtleties about whether one has to use HF or free propagators are not relevant for us, since in the case of a renormalized zero-range interaction, as it is generally used in ultracold atom systems, the HF shift vanishes anyway [10]. However, the fact that we work with zero-temperature propagators corresponding to a fixed density and not to a fixed chemical potential is very important. Among other things, it ensures that the pp-RPA formalism satisfies the Luttinger theorem [12].

It is straight-forward to extend the NSR theory to the imbalanced case, by introducing two different chemical potentials $\mu_{\sigma}(\sigma=\uparrow, \downarrow)$. Unfortunately, as it was already observed by several authors [13 15], this scheme that works nicely in the balanced case fails in the imbalanced case. To be specific, the problem is that near the unitary limit (i.e., for large scattering length: $|a| \rightarrow \infty$ ) one finds in some regions of the phase diagram $\rho_{\uparrow}<\rho_{\downarrow}$ in spite of $\mu_{\uparrow}>\mu_{\downarrow}$.

We suspect that this problem is related to the fact that within the NSR scheme the undressed s.p. Green's functions used to build the ladder diagrams are computed with the same chemical potentials as the corrected Green's functions. In the $T \rightarrow 0$ limit this implies that the ladders are calculated in a system whose Fermi momenta are different from the final ones. As it will be shown in a separate article [16], a finite-temperature formalism that includes the shift of the s.p. energies selfconsistently does not present the pathological behavior of the NSR scheme and reduces to the pp-RPA in the $T \rightarrow 0$ limit. The aim of the present paper is to see what happens at $T=0$ within pp-RPA in the strongly imbalanced case.

\section{PARTICLE-PARTICLE RPA FOR THE STRONGLY IMBALANCED CASE AT ZERO TEMPERATURE}

We consider now a polarized Fermi gas in which the density of $\uparrow$ particles is higher than that of $\downarrow$ particles, $n_{\uparrow}>n_{\downarrow}$. At very strong polarization $P=\left(n_{\uparrow}-n_{\downarrow}\right) /\left(n_{\uparrow}+\right.$ $\left.n_{\downarrow}\right)$, the system remains normal fluid even at zero temperature. It is this case that we want to discuss now. This case includes in particular the polaron, i.e., a single $\downarrow$ particle in a Fermi sea of $\uparrow$ particles, which has recently attracted a lot of attention from theoretical and experimental side [17].

At zero temperature, it is not necessary to use the Matsubara formalism. Instead, one can start from the usual time-ordered s.p. Green's function [9]

$$
G_{0}^{\sigma}(\omega, \mathbf{p})=\frac{\theta\left(k_{F}^{\sigma}-p\right)}{\omega-\epsilon_{\mathbf{p}}-i \eta}+\frac{\theta\left(p-k_{F}^{\sigma}\right)}{\omega-\epsilon_{\mathbf{p}}+i \eta},
$$

where $k_{F}^{\sigma}$ denotes the Fermi momentum of the atoms in spin state $\sigma$. In contrast to the finite-temperature case discussed before, the s.p. energies are in this formalism not measured from the respective Fermi surfaces, i.e., $\epsilon_{\mathbf{p}}=p^{2} /(2 m)$ (throughout the article we use units with $\hbar=1, \hbar$ being the reduced Planck constant).

We mention that this formalism is not equivalent to the zero-temperature limit of the Matsubara formalism: on the one hand, within the Matsubara formalism, the Green's function $G$ is expressed in terms of free Green's functions $G_{0}$ corresponding to the same chemical potentials. On the other hand, in the zero-temperature formalism, $G$ is expressed in terms of $G_{0}$ corresponding to the same densities. The importance of this subtlety in the case of non-perturbative resummations (such as ladder diagrams) will become clearer below.

\section{A. In-medium T matrix}

Let us start by calculating the in-medium $\mathrm{T}$ matrix shown in Fig. 1 within the zero-temperature formalism. As interaction, we consider a contact interaction with coupling constant $g<0$. Then the $\mathrm{T}$ matrix can be written as

$$
\Gamma(\omega, \mathbf{k})=\frac{1}{\frac{1}{g}-J(\omega, \mathbf{k})},
$$

with $J(\omega, \mathbf{k})=J_{\mathrm{hh}}(\omega, \mathbf{k})+J_{\mathrm{pp}}(\omega, \mathbf{k})$ and

$$
\begin{aligned}
& J_{\mathrm{hh}}(\omega, \mathbf{k})=-\int^{\Lambda} \frac{d^{3} p}{(2 \pi)^{3}} \frac{\theta\left(k_{F}^{\uparrow}-p\right) \theta\left(k_{F}^{\downarrow}-|\mathbf{k}-\mathbf{p}|\right)}{\omega-\epsilon_{\mathbf{p}}-\epsilon_{\mathbf{k}-\mathbf{p}}-i \eta}, \\
& J_{\mathrm{pp}}(\omega, \mathbf{k})=\int^{\Lambda} \frac{d^{3} p}{(2 \pi)^{3}} \frac{\theta\left(p-k_{F}^{\uparrow}\right) \theta\left(|\mathbf{k}-\mathbf{p}|-k_{F}^{\downarrow}\right)}{\omega-\epsilon_{\mathbf{p}}-\epsilon_{\mathbf{k}-\mathbf{p}}+i \eta}
\end{aligned}
$$

The subscripts hh and pp denote the contributions of hole-hole and particle-particle propagation, respectively. 
The cutoff $\Lambda$ has been introduced because the momentum integral in $J_{\mathrm{pp}}$ diverges. To be precise, the integration region is defined by $|\mathbf{p}-\mathbf{k} / 2|<\Lambda$, and the upper integration limit in Eqs. (6) and (7) should only be interpreted as a short-hand notation. The usual procedure to deal with the divergence consists in making the coupling constant $g$ dependent on $\Lambda$ and then taking the limit $\Lambda \rightarrow \infty$, keeping the scattering length $a$ constant (which implies $g \rightarrow 0$ ) [10]. The result of this renormalization procedure can be written as follows:

$$
\tilde{\Gamma}(\omega, \mathbf{k})=\frac{1}{\frac{1}{\tilde{g}}-\tilde{J}(\omega, \mathbf{k})},
$$

where $\tilde{g}=4 \pi a / m, \tilde{J}=\tilde{J}_{\mathrm{hh}}+\tilde{J}_{\mathrm{pp}}, \tilde{J}_{\mathrm{hh}}=\lim _{\Lambda \rightarrow \infty} J_{\mathrm{hh}}$, and

$$
\tilde{J}_{\mathrm{pp}}(\omega, \mathbf{k})=\int \frac{d^{3} p}{(2 \pi)^{3}}\left(\frac{\theta\left(p-k_{F}^{\uparrow}\right) \theta\left(|\mathbf{k}-\mathbf{p}|-k_{F}^{\downarrow}\right)}{\omega-\epsilon_{\mathbf{p}}-\epsilon_{\mathbf{k}-\mathbf{p}}+i \eta}+\frac{m}{p^{2}}\right) .
$$

The integrals $\tilde{J}_{\mathrm{hh}}$ and $\tilde{J}_{\mathrm{pp}}$ can be evaluated analytically.

Note that within the RPA scheme we should in principle have started from the HF Green's function instead of the non-interacting one [11]. Consequently, the s.p. energies $\epsilon_{\mathbf{p}}$ in Eqs. (6) - (9) should be replaced by HF energies $\epsilon_{\mathbf{p}}^{\sigma}=p^{2} /(2 m)+g n_{\bar{\sigma}}$, where $\bar{\sigma}$ denotes the spin opposite to $\sigma$. However, as mentioned before, since $g \rightarrow 0$ in the limit $\Lambda \rightarrow \infty$, we shall not bother with this unnecessary complication.

It is useful to analyze in more detail the properties of $\tilde{J}$ and $\tilde{\Gamma}$. We define the variable $q$ corresponding to the on-shell momentum of each atom in the center of mass (c.m.) frame of the pair, via $\omega=q^{2} / m+k^{2} /(4 m)$. In Fig. 3, we show schematically the regions where the imaginary part of $\tilde{\Gamma}$ is non-zero. The circle corresponds to $\omega=\Omega_{F}=\epsilon_{F}^{\uparrow}+\epsilon_{F}^{\downarrow}$, where $\epsilon_{F}^{\sigma}=k_{F}^{\sigma 2} /(2 m)$. This circle separates the regions (1) to (3) (hatched in blue), where the imaginary part comes from $\tilde{J}_{\mathrm{hh}}$, from the regions (4) to (8) (hatched in red), where the imaginary part comes from $\tilde{J}_{\mathrm{pp}}$. On the dashed lines separating the different regions, $\tilde{J}$ has cusps. We see that for $k<k_{F}^{\uparrow}-k_{F}^{\downarrow}$ the hh and pp continua are separated by a region around $\omega=\Omega_{F}$ where the imaginary part vanishes. This is also visible in Fig. 4. where we display the real and imaginary parts of $-\tilde{J}$ for the case $k_{F}^{\downarrow}=k_{F}^{\uparrow} / 2$ and $k=0$. The sharp edges of the hh and pp continua lead to logarithmic singularities in the real part of $\tilde{J}$. Similarly to the original Cooper problem [18], a small attractive interaction $(a<$ 0 ) leads therefore to the existence of two poles in $\tilde{\Gamma}$ (at the energies where $-\tilde{J}$ crosses $-1 / \tilde{g}$ ): one slightly below the edge of the pp continuum, corresponding to a bound pair of two particles, and the other slightly above the edge of the hh continuum, corresponding to a bound pair of two holes. These states are shown schematically as the red and blue lines in Fig. 3. With increasing total momentum $k$, the sharp edges are washed out [regions (2) and (7) in Fig. 3 and the poles disappear.

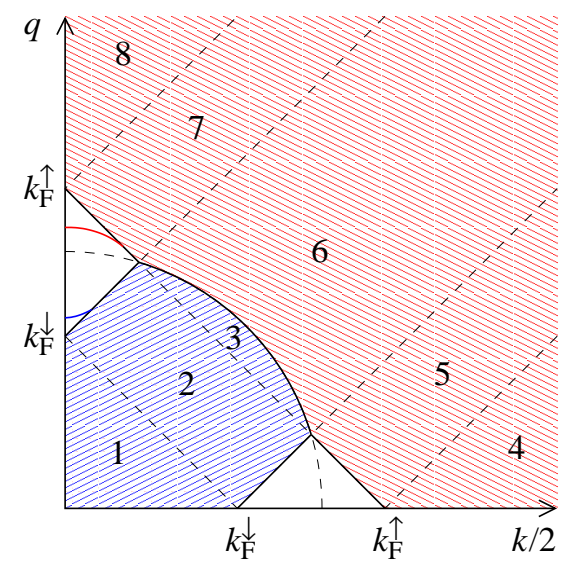

FIG. 3: Regions corresponding to different cases in the calculation of the function $\tilde{J}(\omega, \mathbf{k})$ in terms of the momentum in the center-of-mass frame, $q=\sqrt{m \omega-k^{2} / 4}$, and the total momentum of the pair, $k$. The circle corresponds to $\omega=\Omega_{F}=\epsilon_{F}^{\uparrow}+\epsilon_{F}^{\downarrow}$ and separates the hh continuum (regions 1-3) from the pp continuum (regions 4-8). The solid lines in the triangle between regions 2 and 7 show schematically the positions of the poles $\Omega_{1,2}$ of $\tilde{\Gamma}$.

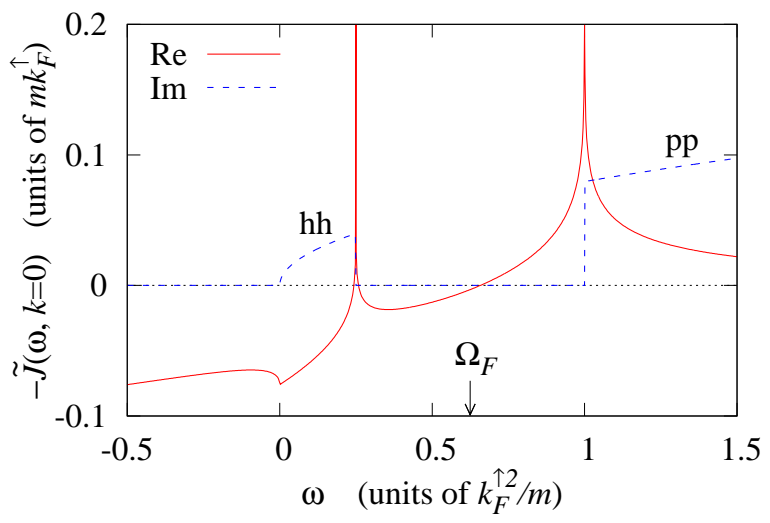

FIG. 4: Real (solid line) and imaginary (dashed line) parts of $-\tilde{J}(\omega, \mathbf{k}=0)$ as function of $\omega$ for $k_{F}^{\downarrow}=k_{F}^{\uparrow} / 2$ (corresponding to a polarization of $P \approx 0.78)$.

As the interaction strength increases, the upper (pp) pole is shifted to lower and lower energy until it reaches $\omega=\Omega_{F}$. As in the NSR case, this indicates the onset of a pairing instability. If one further increases the interaction strength, the pp pole first enters into the energy range $\omega<\Omega_{F}$ of hh excitations, and then, once $1 / \tilde{g}$ drops below the minimum of $-\tilde{J}$, the pole leaves the real $\omega$ axis and becomes complex.

As noticed in Ref. [13] in the NSR framework, it is in the imbalanced case not sufficient to consider only $\mathbf{k}=0$ in the Thouless criterion (1), but the critical temperature is the highest temperature where the $\mathrm{T}$ matrix has a pole at $\omega=\Omega_{F}$ for any value of $\mathbf{k}$, related to a transition towards a FFLO-like phase with oscillating order 
parameter. In the present case we are not interested in the critical temperature ( since we are at $T=0$ ), but in the critical polarization. If we start with a fully polarized system $(P=1)$ and decrease the polarization, the critical polarization $P_{c}$ is reached when the T matrix has for the first time a pole at $\omega=\Omega_{F}$ for any value of $\mathbf{k}$. Actually it is enough to check that

$$
\tilde{J}\left(\Omega_{F}, \mathbf{k}\right)>\frac{1}{\tilde{g}}
$$

is fulfilled for all $\mathbf{k}$.

Experiments in the unitary limit [5], however, show that the transition from the unpaired to the paired phase at low temperature is not of second, but of first order, leading to phase separation between unpaired and paired phases. Our theory does not allow us to check whether a first-order transition appears already at higher polarization than our $P_{c}$, since this requires a calculation of the energy of the system in the paired phase.

\section{B. Self-energy and occupation numbers}

Let us start by writing down the expression for the self-energy diagram shown in Fig. 2(b),

$$
\begin{aligned}
\Sigma^{\sigma}(\omega, \mathbf{p})=-i \int^{\Lambda} \frac{d^{3} p^{\prime}}{(2 \pi)^{3}} \int & \frac{d \omega^{\prime}}{2 \pi} G_{0}^{\bar{\sigma}}\left(\omega^{\prime}, \mathbf{p}^{\prime}\right) \\
& \times \Gamma\left(\omega+\omega^{\prime}, \mathbf{p}+\mathbf{p}^{\prime}\right) .
\end{aligned}
$$

For formal derivations, it will be sometimes convenient to keep the cutoff finite (here, the integration region is defined by $\left.\left|\mathbf{p}-\mathbf{p}^{\prime}\right| / 2<\Lambda\right)$, but in all practical calculations we will use the renormalization procedure, i.e., replace $\Gamma$ by $\tilde{\Gamma}$ and let the cutoff $\Lambda$ go to infinity.

To evaluate Eq. (11), it is helpful to split $\Gamma$ into the bare interaction $g$ (which vanishes in the limit $\Lambda \rightarrow \infty$ ) and forward and backward going parts,

$$
\Gamma=g+\Gamma_{\mathrm{pp}}+\Gamma_{\mathrm{hh}}
$$

Note that in $\Gamma$ the pp and hh channels are summed up together, so that $\Gamma_{\mathrm{pp}}$ contains also contributions from hh propagation and vice versa, as illustrated in Fig. 5. The separation into forward and backward going parts can be achieved with the help of dispersion relations:

$$
\begin{aligned}
& \Gamma_{\mathrm{pp}}(\omega, \mathbf{k})=-\int_{\Omega_{F}}^{\infty} \frac{d \omega^{\prime}}{\pi} \frac{\operatorname{Im} \Gamma\left(\omega^{\prime}, \mathbf{k}\right)}{\omega-\omega^{\prime}+i \eta} \\
& \Gamma_{\mathrm{hh}}(\omega, \mathbf{k})=\int_{-\infty}^{\Omega_{F}} \frac{d \omega^{\prime}}{\pi} \frac{\operatorname{Im} \Gamma\left(\omega^{\prime}, \mathbf{k}\right)}{\omega-\omega^{\prime}-i \eta}
\end{aligned}
$$

The imaginary parts in the numerators are meant to include also the contribution of possible poles of $\Gamma$ at $\omega=\Omega_{i}$, i.e.,

$$
\operatorname{Im} \Gamma(\omega, \mathbf{k})=\frac{\operatorname{Im} J}{\left|\frac{1}{g}-J\right|^{2}}-\pi \sum_{i} S_{i}(\mathbf{k}) \delta\left(\omega-\Omega_{i}(\mathbf{k})\right)
$$

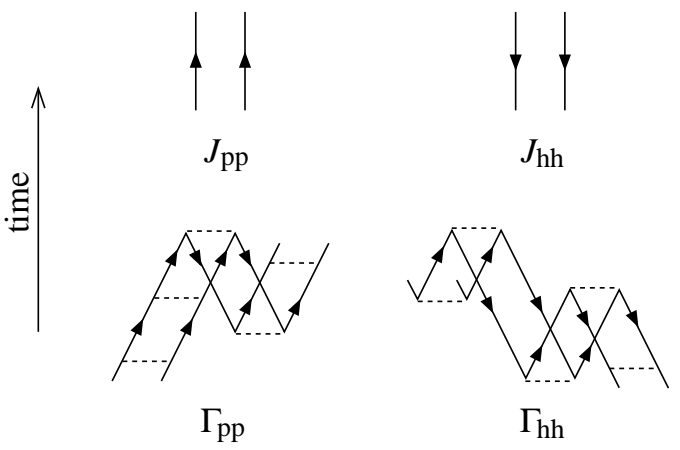

FIG. 5: Diagrammatic illustration of forward and backward going parts: free pp and hh propagators $J_{\mathrm{pp}}$ and $J_{\mathrm{hh}}$ (top), typical contributions to the forward and backward going parts of the $\mathrm{T}$ matrix, $\Gamma_{\mathrm{pp}}$ and $\Gamma_{\mathrm{hh}}$ (bottom).

where the strengths of the poles are given by $S_{i}=$ $1 /(d J / d \omega)_{\Omega_{i}} \operatorname{sgn}\left(\Omega_{F}-\Omega_{i}\right)$. The self-energy can now also be written as a sum of the energy-independent HF term and forward and backward going parts,

$$
\Sigma^{\sigma}=g n_{\bar{\sigma}}+\Sigma_{\mathrm{pp}}^{\sigma}+\Sigma_{\mathrm{hh}}^{\sigma}
$$

with

$$
\begin{aligned}
& \Sigma_{\mathrm{pp}}^{\sigma}(\omega, \mathbf{p})=\int^{\Lambda} \frac{d^{3} p^{\prime}}{(2 \pi)^{3}} \theta\left(k_{F}^{\bar{\sigma}}-p^{\prime}\right) \Gamma_{\mathrm{pp}}\left(\omega+\epsilon_{\mathbf{p}^{\prime}}, \mathbf{p}+\mathbf{p}^{\prime}\right), \\
& \Sigma_{\mathrm{hh}}^{\sigma}(\omega, \mathbf{p})=-\int^{\Lambda} \frac{d^{3} p^{\prime}}{(2 \pi)^{3}} \theta\left(p^{\prime}-k_{F}^{\bar{\sigma}}\right) \Gamma_{\mathrm{hh}}\left(\omega+\epsilon_{\mathbf{p}^{\prime}}, \mathbf{p}+\mathbf{p}^{\prime}\right) .
\end{aligned}
$$

In the RPA scheme, the HF term $g n_{\bar{\sigma}}$ must be removed from $\Sigma$ since it is already contained in the HF s.p. energies $\epsilon_{\mathbf{p}}^{\sigma}$, but in the limit $\Lambda \rightarrow \infty$ it vanishes anyway.

The aim of this section is the calculation of the occupation numbers $n_{\mathbf{p}}^{\sigma}$. In terms of the zero-temperature Green's function, they can be obtained from [9]

$$
n_{\mathbf{p}}^{\sigma}=-i \int \frac{d \omega}{2 \pi} e^{i \omega \eta} G^{\sigma}(\omega, \mathbf{p}) .
$$

If one keeps, as in Eq. (3), only the first-order term of the Dyson equation, one readily obtains

$$
\begin{aligned}
n_{\mathbf{p}}^{\sigma}=\theta\left(k_{F}^{\sigma}-\right. & p)\left(1-i \int \frac{d \omega}{2 \pi} \frac{\Sigma^{\sigma}(\omega, \mathbf{p})}{\left(\omega-\epsilon_{\mathbf{p}}-i \eta\right)^{2}}\right) \\
- & i \theta\left(p-k_{F}^{\sigma}\right) \int \frac{d \omega}{2 \pi} \frac{\Sigma^{\sigma}(\omega, \mathbf{p})}{\left(\omega-\epsilon_{\mathbf{p}}+i \eta\right)^{2}} .
\end{aligned}
$$

With the help of the residue theorem, this can be written as

$$
\begin{aligned}
n_{\mathbf{p}}^{\sigma}=\theta\left(k_{F}^{\sigma}-p\right) & \left(1+\left.\frac{d}{d \omega} \Sigma_{\mathrm{pp}}^{\sigma}(\omega, \mathbf{p})\right|_{\omega=\epsilon_{\mathbf{p}}}\right) \\
& -\left.\theta\left(p-k_{F}^{\sigma}\right) \frac{d}{d \omega} \Sigma_{\mathrm{hh}}^{\sigma}(\omega, \mathbf{p})\right|_{\omega=\epsilon_{\mathbf{p}}} .
\end{aligned}
$$




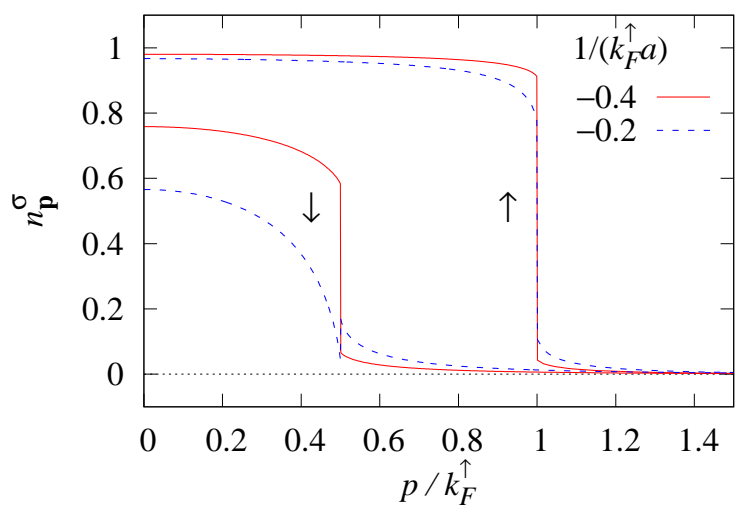

FIG. 6: Occupation numbers of majority ( $\uparrow$, upper curves) and minority ( $\downarrow$, lower curves) particles as function of momentum, for $k_{F}^{\downarrow}=k_{F}^{\uparrow} / 2$ and two different interaction strengths $1 /\left(k_{F}^{\uparrow} a\right)=-0.4$ (solid lines) and -0.2 (dashed lines).

One sees that in the case $p>k_{F}^{\sigma}$ only backward going ladders contribute to the occupation numbers (however, remember the remark after Eq. (12)). Likewise, in the case $p<k_{F}^{\sigma}$ only forward going ladders contribute. For the numerical evaluation it is convenient to transform the expressions for the occupation numbers with the help of Eqs. (13) - (18). For $p>k_{F}^{\sigma}$, one gets

$$
n_{\mathbf{p}}^{\sigma}=-\int^{\Lambda} \frac{d^{3} k}{(2 \pi)^{3}} \int_{-\infty}^{\Omega_{F}} \frac{d \omega}{\pi} \frac{\theta\left(|\mathbf{k}-\mathbf{p}|-k_{F}^{\bar{\sigma}}\right)}{\left(\omega-\epsilon_{\mathbf{p}}-\epsilon_{\mathbf{k}-\mathbf{p}}\right)^{2}} \operatorname{Im} \Gamma(\omega, \mathbf{k}) .
$$

Note that the denominator in Eq. (22) cannot become zero because of the upper limit of the $\omega$ integral, the theta function, and the condition $p>k_{F}^{\sigma}$. Analogously, one obtains for $p<k_{F}^{\sigma}$

$$
n_{\mathbf{p}}^{\sigma}=1+\int \frac{d^{3} k}{(2 \pi)^{3}} \int_{\Omega_{F}}^{\infty} \frac{d \omega}{\pi} \frac{\theta\left(k_{F}^{\bar{\sigma}}-|\mathbf{k}-\mathbf{p}|\right)}{\left(\omega-\epsilon_{\mathbf{p}}-\epsilon_{\mathbf{k}-\mathbf{p}}\right)^{2}} \operatorname{Im} \Gamma(\omega, \mathbf{k}) .
$$

In this integral, the cutoff can be omitted since the relative momentum of the two particles is anyway limited to $|\mathbf{k} / 2-\mathbf{p}| \leq\left(k_{F}^{\uparrow}+k_{F}^{\downarrow}\right) / 2$ because of the condition $p<k_{F}^{\sigma}$ and the theta function.

In practice, as mentioned before, we replace $\Gamma$ by $\tilde{\Gamma}$ and let the cutoff $\Lambda$ go to infinity. The angular integrals in Eqs. (22) and (23) can be evaluated analytically. The integrals over $\omega$ are split into pole and continuum contributions. The contributions of the delta functions in $\operatorname{Im} \Gamma$, see Eq. (15), are of course included analytically, while the continuum contributions are computed numerically. The remaining integrals over $k$ are done numerically, too.

As an example, we show in Fig. 6] the occupation numbers for $k_{F}^{\downarrow}=k_{F}^{\uparrow} / 2$ for two different interaction strengths. We see that the correlations reduce $n_{\mathbf{p}}^{\sigma}$ below $k_{F}^{\sigma}$ and generate non-vanishing $n_{\mathbf{p}}^{\sigma}$ above $k_{F}^{\sigma}$. This is effect is much stronger for the minority $(\downarrow)$ particles than for the majority $(\uparrow)$ particles. For $1 /\left(k_{F}^{\uparrow} a\right)=-0.4$, the occupation numbers look reasonable, but in the more strongly interacting case $1 /\left(k_{F}^{\uparrow} a\right)=-0.2$ the jump in the $\downarrow$ occupation numbers has the wrong sign. If we increase the interaction further, the $\downarrow$ occupation numbers even become negative below the Fermi surface. This pathological behavior is a consequence of Eq. (3), where the correlations are treated perturbatively by truncating the Dyson equation at first order.

Actually, from Eq. (21) one sees that the jump of $n_{\mathbf{p}}^{\sigma}$ is given by

$$
n_{|\mathbf{p}| \rightarrow k_{F}^{\sigma-}}^{\sigma}-n_{|\mathbf{p}| \rightarrow k_{F}^{\sigma+}}^{\sigma}=1+\left.\frac{d}{d \omega} \Sigma^{\sigma}\left(\omega, k_{F}^{\sigma}\right)\right|_{\omega=\epsilon_{F}^{\sigma}} .
$$

This has to be compared with the exact result

$$
n_{|\mathbf{p}| \rightarrow k_{F}^{\sigma-}}^{\sigma}-n_{|\mathbf{p}| \rightarrow k_{F}^{\sigma+}}^{\sigma}=Z_{k_{F}^{\sigma}}^{\sigma}
$$

where $Z_{\mathbf{p}}^{\sigma}=1 /\left[1-d \Sigma^{\sigma}(\omega, \mathbf{p}) /\left.d \omega\right|_{\omega=\epsilon_{\mathbf{p}}^{\sigma *}}\right]$ is the quasiparticle residue of the s.p. Green's function, with the quasiparticle energy $\epsilon_{\mathbf{p}}^{\sigma *}=\epsilon_{\mathbf{p}}^{\sigma}+\Sigma^{\sigma}\left(\epsilon_{\mathbf{p}}^{\sigma *}, \mathbf{p}\right)$ [19]. Equations (24) and (25) agree to leading order in $\Sigma^{\sigma}$ and the change of sign in Eq. (24) for $d \Sigma^{\sigma} / d \omega<-1$ is just a consequence of the breakdown of the expansion $1 /\left(1-d \Sigma^{\sigma} / d \omega\right) \approx 1+d \Sigma^{\sigma} / d \omega+\ldots$

\section{Luttinger theorem}

The Luttinger theorem [12] states that the correlated occupation numbers $n_{\mathbf{p}}^{\sigma}$ have their discontinuity still at $k_{F}^{\sigma}=\left(6 \pi^{2} n_{\sigma}\right)^{1 / 3}$. In other words, if we define

$$
\begin{aligned}
& \delta n_{\mathrm{h}}^{\sigma}=\int \frac{d^{3} p}{(2 \pi)^{3}} \theta\left(k_{F}^{\sigma}-p\right)\left(n_{\mathbf{p}}^{\sigma}-1\right) \\
& \delta n_{\mathrm{p}}^{\sigma}=\int \frac{d^{3} p}{(2 \pi)^{3}} \theta\left(p-k_{F}^{\sigma}\right) n_{\mathbf{p}}^{\sigma}
\end{aligned}
$$

the occupation numbers have to satisfy $\delta n_{\mathrm{h}}^{\sigma}+\delta n_{\mathrm{p}}^{\sigma}=0$.

Using the equations of the preceding subsections and the analytic properties of the different functions in the complex plane, one can show after some transformations that

$$
\begin{aligned}
\delta n_{\mathrm{h}}^{\sigma} & =\int \frac{d^{3} k}{(2 \pi)^{3}} \int \frac{d \omega}{2 \pi} \operatorname{Im}\left(\Gamma(\omega, \mathbf{k}) \frac{d}{d \omega} J_{\mathrm{hh}}(\omega, \mathbf{k})\right), \\
\delta n_{\mathrm{p}}^{\sigma} & =\int \frac{d^{3} k}{(2 \pi)^{3}} \int \frac{d \omega}{2 \pi} \operatorname{Im}\left(\Gamma(\omega, \mathbf{k}) \frac{d}{d \omega} J_{\mathrm{pp}}(\omega, \mathbf{k})\right) .
\end{aligned}
$$

In the derivation of Eq. (29), we made use of the cutoff regularization, which ensures that $J_{\mathrm{pp}}$ falls off like $1 / \omega$ for $\omega \rightarrow \infty$. Interestingly, we see that $\delta n_{\mathrm{h}}^{\sigma}$ and $\delta n_{\mathrm{p}}^{\sigma}$ are independent of $\sigma$.

In order to show that the Luttinger theorem is satis- 
fied, we add Eqs. (28) and (29):

$$
\begin{aligned}
\delta n_{\mathrm{h}}^{\sigma}+\delta n_{\mathrm{p}}^{\sigma} & =\int \frac{d^{3} k}{(2 \pi)^{3}} \int \frac{d \omega}{2 \pi} \operatorname{Im}\left(\Gamma \frac{d J}{d \omega}\right) \\
& =-\int \frac{d^{3} k}{(2 \pi)^{3}} \int \frac{d \omega}{2 \pi} \frac{d}{d \omega} \operatorname{Im} \log (1-g J) \\
& =0 .
\end{aligned}
$$

Again we have used the cutoff regularization which ensures that $J \rightarrow 0$ for $\omega \rightarrow \infty$.

The proof can also be carried out with the renormalized functions $\tilde{J}$ and $\tilde{\Gamma}$, but it is more cumbersome in that case. If we integrate numerically our occupation numbers, which are obtained with the renormalized functions, the Luttinger theorem is satisfied to a precision of $\sim 10^{-4}$.

In nuclear physics, the fact that pp-RPA does not modify the sum of particle- and hole occupation numbers in finite nuclei (having discrete particle- and hole levels) has been known for many years, see Ref. [20]. Recently, the pp-RPA formalism has been also applied to the case of Bose-Fermi mixtures, and there it was also found that it respects the Luttinger theorem for the Fermion occupation numbers [21].

\section{Energy density and chemical potentials}

While in the standard NSR approach the corrections to the densities $n_{\sigma}$ are calculated for fixed chemical potentials $\mu_{\sigma}$, we have just seen that in the zero-temperature formalism there are no corrections to the densities due to correlations. However, there are corrections to the chemical potentials, so that in the end the relationships between $\mu_{\sigma}$ and $n_{\sigma}$ are changed in the zero-temperature formalism, too. If one did a strictly perturbative expansion, i.e., without resummation of ladder diagrams, the difference between the relationships $n_{\sigma}\left(\mu_{\uparrow}, \mu_{\downarrow}\right)$ or, vice versa, $\mu_{\sigma}\left(n_{\uparrow}, n_{\downarrow}\right)$ obtained in the two formalisms should be of higher order than the expansion [19].

Here we will calculate the chemical potentials from the energy density $\mathcal{E}$ :

$$
\mu_{\sigma}=\frac{\partial \mathcal{E}}{\partial n_{\sigma}} .
$$

The correlation energy density, $\delta \mathcal{E}=\mathcal{E}-\mathcal{E}_{0}$, where $\mathcal{E}_{0}=\left(k_{F}^{\uparrow 5}+k_{F}^{\downarrow 5}\right) /\left(20 \pi^{2} m\right)+g n_{\uparrow} n_{\downarrow}$ is the HF energy density of the uncorrelated system, can be derived from the following general formula [9]

$$
\delta \mathcal{E}=-\frac{i}{2} \int_{0}^{1} \frac{d \lambda}{\lambda} \int \frac{d^{3} p}{(2 \pi)^{3}} \int \frac{d \omega}{2 \pi} e^{i \omega \eta}\left(\omega-\epsilon_{\mathbf{p}}\right)\left(G_{\lambda}^{\uparrow}+G_{\lambda}^{\downarrow}\right) .
$$

In Eq. (32), $G_{\lambda}^{\sigma}$ denotes the Green's function calculated with coupling constant $\lambda g$ instead of $g$. More precisely, since RPA theory is built on top of the HF ground state (this is why $\mathcal{E}_{0}$ is not the non-interacting but the HF energy density), the coupling constant entering the HF field must not be multiplied by $\lambda$. Anyway, this detail is not important since the HF field vanishes in the limit $\Lambda \rightarrow \infty$.

By inserting Eqs. (3) and (16) (without the HF term $\left.g n_{\bar{\sigma}}\right)$ into Eq. (32), one readily obtains

$$
\begin{array}{r}
\delta \mathcal{E}=\frac{1}{2} \int_{0}^{1} \frac{d \lambda}{\lambda} \int \frac{d^{3} p}{(2 \pi)^{3}} \sum_{\sigma}\left(-\theta\left(p-k_{F}^{\sigma}\right) \Sigma_{\lambda \mathrm{hh}}\left(\epsilon_{\mathbf{p}}, \mathbf{p}\right)\right. \\
\left.+\theta\left(k_{F}^{\sigma}-p\right) \Sigma_{\lambda \mathrm{pp}}^{\sigma}\left(\epsilon_{\mathbf{p}}, \mathbf{p}\right)\right)
\end{array}
$$

Using the expressions given in Secs. IIA and IIB and exploiting the analytical properties of the functions in the complex plane, one can show that this expression is equal to

$$
\delta \mathcal{E}=-\int_{0}^{1} \frac{d \lambda}{\lambda} \int \frac{d^{3} k}{(2 \pi)^{3}} \int_{-\infty}^{\Omega_{F}} \frac{d \omega}{\pi} \operatorname{Im}\left(\left(\Gamma_{\lambda}-\lambda g\right) J\right) .
$$

Let us now look at the integral over $\lambda$ :

$$
\int_{0}^{1} \frac{d \lambda}{\lambda} \Gamma_{\lambda}=\int_{0}^{1} d \lambda \frac{1}{\frac{1}{g}-\lambda J}=-\frac{1}{J} \log (1-g J) .
$$

Some care has to be taken in the presence of poles in $\Gamma$, since in this case the argument of the logarithm can become negative. In Fig. 4, this corresponds to the region between the hh continuum and the blue line, and between the red line and the pp continuum. In these regions, we have $\operatorname{Im} \log (1-g J)=-\pi$. Now Eq. (34) becomes

$$
\delta \mathcal{E}=\int \frac{d^{3} k}{(2 \pi)^{3}} \int_{-\infty}^{\Omega_{F}} \frac{d \omega}{\pi} \operatorname{Im}(\log (1-g J)+g J) .
$$

Although we have assumed in our derivation that $J$ is regularized with a cutoff $\Lambda$, we can now take the limit $\Lambda \rightarrow \infty$. In this limit, the contribution of the last term of Eq. (36) vanishes, and we are left with the following compact formula for the correlation energy:

$$
\delta \mathcal{E}=\int \frac{d^{3} k}{(2 \pi)^{3}} \int_{-\infty}^{\Omega_{F}} \frac{d \omega}{\pi} \operatorname{Im} \log \left(\tilde{J}-\frac{1}{\tilde{g}}\right) .
$$

In our calculation of the energy density, the integrals over $k$ and $\omega$ are done numerically (except for the $\omega$ integral of the pole contribution). We have not attempted to derive formulas for the chemical potentials and we compute them by numerically differentiating the energy density.

In Fig. 7, we show the chemical potentials $\mu_{\uparrow}$ and $\mu_{\downarrow}$ as functions of the polarization $P$ for fixed $n_{\uparrow}$ for different interaction strengths. For each interaction strength, the polarization is varied over the range in which the condition (10) is fulfilled, i.e., $P>P_{c}$. As one would expect, the chemical potential of the minority species, $\mu_{\downarrow}$, is much more strongly lowered by interactions than that of the majority species, $\mu_{\uparrow}$. Actually, already within 


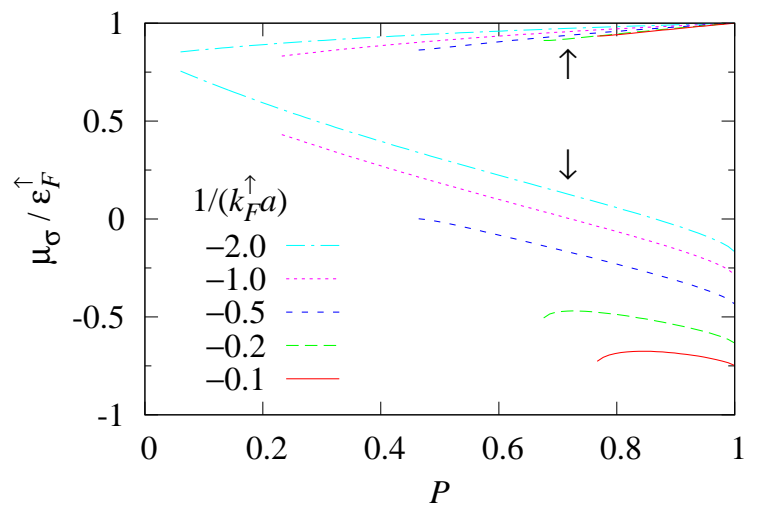

FIG. 7: Chemical potentials of the majority ( $\uparrow$, upper curves) and minority ( $\downarrow$, lower curves) particles as functions of the polarization for different interaction strengths from $1 /\left(k_{F}^{\uparrow} a\right)=$ -2 (dash-dotted lines) to -0.1 (solid lines).

the "Hartree approximation" $\left(\mu_{\sigma}=\epsilon_{F}^{\sigma}+\tilde{g} n_{\bar{\sigma}}\right)$ it is like that $^{1}$. At the two strongest interactions $1 /\left(k_{F}^{\uparrow} a\right)=-0.2$ and -0.1 , one observes that $\mu_{\downarrow}$ increases with decreasing $n_{\downarrow}$ at polarizations $P<0.73$ and $P<0.85$, respectively.

One could be tempted to say that $\partial \mu_{\downarrow} / \partial n_{\downarrow}<0$ indicates an instability towards phase separation into a more and a less polarized phase (first-order phase transition). This would be nice, because experimentally it is found that the system separates into a polarized and an unpolarized phase below some critical polarization [5]. However, the agreement would be purely qualitative since the critical polarization observed in the experiment is much lower than ours (e.g., in the unitary limit, it is about 0.4 5 ). Furthermore one should remember that at the polarizations where we find $\partial \mu_{\downarrow} / \partial n_{\downarrow}<0$, the truncation of the Dyson equation to first order in $\Sigma$ gives the wrong sign of the jump in the occupation numbers $n_{\mathbf{p}}^{\downarrow}$, cf. Fig. 6] If one discards those cases where the jump of $n_{\mathbf{p}}^{\downarrow}$ has the wrong sign, one should only consider polarizations $P>0.81$ for $1 /\left(k_{F}^{\uparrow} a\right)=-0.2$ and $P>0.94$ for $1 /\left(k_{F}^{\uparrow} a\right)=-0.1$, respectively.

Finally, let us discuss the limit $P \rightarrow 1$ (i.e., $n_{\downarrow} \rightarrow$ 0 ), corresponding to the polaron. In Fig. 8 , we display our results (pp-RPA) for the polaron energy, which is equal to $\mu_{\downarrow}$ in the limit of $n_{\downarrow} \rightarrow 0$, as a function of the interaction strength. These results can be compared with the Hartree approximation $\mu_{\downarrow}=\tilde{g} n_{\uparrow}$ and the results of a calculation by Combescot et al. 22], which are in

\footnotetext{
${ }^{1}$ As discussed before, the true HF shift $g n_{\bar{\sigma}}$ vanishes for the regularized contact interaction in the limit $\Lambda \rightarrow \infty$. However, in the weak-coupling limit, $\tilde{\Gamma}$ can be approximated by $\tilde{g}$ and this leads to a constant shift $\tilde{g} n_{\sigma}$ which is usually referred to as Hartree shift (there is no exchange term because the interaction acts only between particles of opposite spin).
}

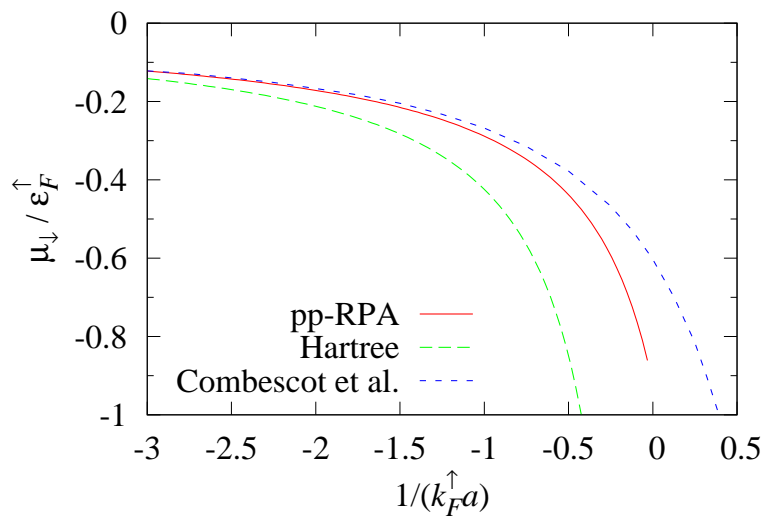

FIG. 8: Polaron chemical potential obtained within pp-RPA (solid line), compared with the Hartree approximation (cf. footnote) $\tilde{g} n_{\uparrow}$ (long dashes) and with the results of Ref. 22] (short dashes).

very good agreement with quantum Monte-Carlo (QMC) results [23, 24]. The calculation of Ref. 22] is actually also based on the T matrix, but the polaron energy is defined in a completely different way as the pole of $G^{\downarrow}$ in which the self-energy is summed to all orders. We see that our results represent a considerable improvement over the Hartree approximation and stay very close to the results of Ref. [22] up to $1 /\left(k_{F}^{\uparrow} a\right) \sim-1$.

\section{DISCUSSION, CONCLUSION, OUTLOOK}

In this work we considered the pairing aspects of strongly polarized Fermi gases. We worked at zero temperature within the RPA theory in the particle-particle (pp) channel (summation of ladders) and discussed in detail similarities and differences with the NSR scheme. The latter gave in the recent past some pathological results when applied to polarized Fermi gases near the unitary limit [13 15]. These might be related to the fact that within the NSR scheme the undressed Green's functions building the ladder diagrams are calculated with the same chemical potential as the final one. This is the main difference from the zero-temperature pp-RPA formalism, where the Green's functions depend on $k_{F}$ and not on $\mu$.

Using the pp-RPA, we have calculated the correction to the occupation numbers. In particular, we have shown that the occupation numbers satisfy the Luttinger theorem. Actually we had already shown this in a similar scenario for interacting bosons and fermions in a mixture [21]. But we have also seen that the approach breaks down when the correlations become too strong (i.e., when the attraction becomes too strong or the polarization becomes too small). This problem stems from the fact that for consistency with the RPA formalism the self-energy in the Dyson equation can only be treated perturbatively 
to first order (by the way, this truncation is also made in the original NSR scheme). It seems very unlikely that the nice properties of the pp-RPA, such as the fact that it satisfies the Luttinger theorem exactly, remain valid if the Dyson series is summed up (of course the error may be quantitatively small).

We have also computed the corrections to the chemical potentials. Of particular interest is the limit of extreme asymmetry, corresponding to the polaron, i.e., a single $\downarrow$ particle in a Fermi sea of $\uparrow$ particles. In this limit, we found good agreement with the results by Combescot et al. 22] up to a certain strong attraction below the unitary limit, see Fig. 8 . Again, this is a limitation due to the truncation of the Dyson equation at first order.

The calculations presented here were all based on the zero-temperature formalism. It is therefore not obvious how one can generalize them to finite temperature. As it will be shown in a separate article [16], it is possible to recover the results of the present work in the $T \rightarrow 0$ limit of the finite-temperature formalism if one includes the shift of the quasiparticle energies self-consistently into the Green's functions $G_{0}$ that build the ladder diagrams and the self-energy.

On a quantitative level, the critical polarization we obtain with our approach is (at least in the unitary limit) much larger than the one found experimentally [5] and in QMC calculations [23, 24]. This calls for an improvement of the RPA approach. Several lines are open. One obvious drawback of the RPA is that it calculates groundstate correlations but the ingredients to RPA, e.g., the occupation numbers, are given by the non-correlated ones, cf. the step functions in Eqs. (6) and (7). Since we have calculated the correlated occupation numbers, a natural idea would be to insert those in an improved RPA and iterate to self-consistency. This would probably wash out the discontinuities in $J$ (cf. Fig. (4) and thereby reduce the critical polarization. Such a procedure is often applied in nuclear physics and called there "renormalized RPA" 26, 27]. A still farther reaching (but numerically difficult) improvement of RPA is the so-called "selfconsistent RPA", in which not only occupation numbers are included self-consistently, but also vertex corrections [27 29].

Another probably important thing would be the inclusion of screening of the interaction, which is known to reduce the gap in the balanced case (Gor'kov-MelikBarkhudarov correction [25]) and which therefore could also reduce the critical polarization. However, it is not clear how to include these particle-hole effects consistently into the particle-particle ladders.
[1] D.M. Eagles, Phys. Rev. 186, 456 (1969).

[2] A.J. Leggett, J. Phys. (Paris) 41, C7-19 (1980).

[3] P. Nozières and S. Schmitt-Rink, J. Low Temp. Phys. 59, 195 (1985).

[4] M. Greiner, C.A. Regal, and D.S. Jin, Nature 426, 537 (2003).

[5] Y. Shin, C.H. Schunck, A. Schirotzek, and W. Ketterle, Nature 451, 689 (2008).

[6] P. Fulde and R.A. Ferrell, Phys. Rev. 135, A550 (1964).

[7] A.I. Larkin and Yu.N. Ovchinnikov, Sov. Phys. JETP 20, 762 (1965).

[8] M. Punk, P.T. Dumitrescu, W. Zwerger, Phys. Rev. A 80, 053605 (2009).

[9] A.L. Fetter and J.D. Walecka, Quantum Theory of ManyParticle Systems (McGraw-Hill, New York, 1971).

[10] A. Perali, P. Pieri, G.C. Strinati, and C. Castellani, Phys. Rev. B 66, 024510

[11] P. Ring and P. Schuck, The Nuclear Many-Body Problem (Springer-Verlag, Berlin, 1980)

[12] J.M. Luttinger, Phys. Rev. 119, 1153 (1960).

[13] X.-J. Liu, H. Hu, Europhys. Lett. 75, 364 (2006).

[14] M.M. Parish, F.M. Marchetti, A. Lamacraft, and B.D. Simons, Nature Phys. 3, 124 (2007).

[15] T. Kashimura, R. Watanabe, and Y. Ohashi, J. Low Temp. Phys. 171, 355 (2013).

[16] P.-A. Pantel, D. Davesne, and M. Urban, in preparation.

[17] F. Chevy and C. Mora, Rep. Prog Phys. 73112401
(2010).

[18] L.N. Cooper, Phys. Rev. 104, 1189 (1956).

[19] W.H. Dickhoff and D. Van Neck, Many-Body Theory Exposed! (World Scientific, New Jersey, 2008).

[20] A. Bouyssy and N. Vinh Mau, Nucl. Phys. A 229, 1 (1974).

[21] T. Sogo, P. Schuck, and M. Urban, Phys. Rev. A 88, 023613 (2013).

[22] R. Combescot, A. Recati, C. Lobo, and F. Chevy, Phys. Rev. Lett. 98, 180402 (2007).

[23] C. Lobo, A. Recati, S. Giorgini, and S. Stringari, Phys. Rev. Lett. 97, 200403 (2006).

[24] S. Pilati and S. Giorgini, Phys. Rev. Lett. 100, 030401 (2008).

[25] L.P. Gor'kov and T.K. Melik-Barkhudarov, J. Exp. Theor. Phys. (USSR) 40, 1452 (1961) [translation: Sov. Phys. JETP 13, 1018 (1961)].

[26] F. Catara, G. Piccitto, M. Sambataro, N. Van Giai, Phys. Rev. B 54, 17536 (1996).

[27] D.S. Delion, P. Schuck, and J. Dukelsky, Phys. Rev. C 72, 064305 (2005).

[28] J.G. Hirsch, A. Mariano, J. Dukelsky, and P. Schuck, Ann. Phys. (N.Y.) 296, 187 (2002).

[29] A. Storozhenko, P. Schuck, J. Dukelsky, G. Röpke, and A. Vdovin, Ann. Phys. (N.Y.) 307, 308 (2003). 